Probability, Networks and Algorithms periods and service interruptions

O. Kella, O.J. Boxma, M.R.H. Mandjes 
Centrum voor Wiskunde en Informatica (CWI) is the national research institute for Mathematics and Computer Science. It is sponsored by the Netherlands Organisation for Scientific Research (NWO).

CWI is a founding member of ERCIM, the European Research Consortium for Informatics and Mathematics.

CWI's research has a theme-oriented structure and is grouped into four clusters. Listed below are the names of the clusters and in parentheses their acronyms.

\section{Probability, Networks and Algorithms (PNA)}

Software Engineering (SEN)

Modelling, Analysis and Simulation (MAS)

Information Systems (INS)

Copyright (C) 2008, Stichting Centrum voor Wiskunde en Informatica

P.O. Box 94079, 1090 GB Amsterdam (NL)

Kruislaan 413, 1098 SJ Amsterdam (NL)

Telephone +31205929333

Telefax +31205924199

ISSN 1386-3711 


\title{
On Levy-driven vacation models with correlated busy periods and service interruptions
}

\begin{abstract}
This paper considers queues with server vacations, but departs from the traditional setting in two ways: (i) the queueing model is driven by Levy processes rather than just compound Poisson processes; (ii) the vacation lengths depend on the length of the server's preceding busy period. Regarding the former point: the Levy process active during the busy period is assumed to have no negative jumps, whereas the Levy process active during the vacation is a subordinator. Regarding the latter point: where in a previous study [3] the durations of the vacations were positively correlated with the length of the preceding busy period, we now introduce a dependence structure that may give rise to both positive and negative correlations. We analyze the steady-state workload of the resulting queueing (or: storage) system, by first considering the queue at embedded epochs (viz. the beginnings of busy periods). We show that this embedded process does not always have a proper stationary distribution, due to the fact that there may occur an infinite number of busy-vacation cycles in a finite time interval; we specify conditions under which the embedded process is recurrent. Fortunately, irrespective of whether the embedded process has a stationary distribution, the steady-state workload of the continuous-time storage process can be determined. In addition a number of ramifications are presented. The theory is illustrated by several examples.
\end{abstract}

2000 Mathematics Subject Classification: 60K25

Keywords and Phrases: Levy processes, storage processes, vacation models

Note: The research of O. Boxma and M. Mandjes has been partly funded by the Dutch Bsik/BRICKS (Basic Research in Informatics for Creating the Knowledge Society) project. O. Kella's research is partially supported by grant No. 964/06 from the Israel Science Foundation and the Vigevani Chair in Statistics. 



\title{
On Lévy-driven vacation models with correlated busy periods and service interruptions
}

\author{
Offer Kella* Onno Boxma ${ }^{\dagger} \quad$ Michel Mandjes ${ }^{\ddagger}$
}

\begin{abstract}
This paper considers queues with server vacations, but departs from the traditional setting in two ways: (i) the queueing model is driven by Lévy processes rather than just compound Poisson processes; (ii) the vacation lengths depend on the length of the server's preceding busy period. Regarding the former point: the Lévy process active during the busy period is assumed to have no negative jumps, whereas the Lévy process active during the vacation is a subordinator. Regarding the latter point: where in a previous study [3] the durations of the vacations were positively correlated with the length of the preceding busy period, we now introduce a dependence structure that may give rise to both positive and negative correlations.

We analyze the steady-state workload of the resulting queueing (or: storage) system, by first considering the queue at embedded epochs (viz. the beginnings of busy periods). We show that this embedded process does not always have a proper stationary distribution, due to the fact that there may occur an infinite number of busy-vacation cycles in a finite time interval; we specify conditions under which the embedded process is recurrent. Fortunately, irrespective of whether the embedded process has a stationary distribution, the steady-state workload of the continuous-time storage process can be determined. In addition a number of ramifications are presented. The theory is illustrated by several examples.
\end{abstract}

Keywords: Lévy process, storage process, busy period, queues with server vacations AMS Subject Classification: Primary 60K05; Secondary 60K25

${ }^{*}$ Department of Statistics, The Hebrew University of Jerusalem, Mount Scopus, Jerusalem 91905, Israel; part of this work was done while OK was at Eurandom, P.O. Box 513, 5600 MB Eindhoven, The Netherlands (Offer.Kella@huji.ac.il).

${ }^{\dagger}$ EURANDOM and Department of Mathematics and Computer Science, Eindhoven University of Technology, P.O. Box 513, 5600 MB Eindhoven, The Netherlands (boxma@win.tue.nl).

${ }^{\ddagger}$ Korteweg-de Vries Institute for Mathematics, The University of Amsterdam, Plantage Muidergracht 24; 1018 TV Amsterdam, The Netherlands. MM is also affiliated to CWI, P.O. Box 94079, 1090 GB Amsterdam, The Netherlands and EURANDOM, P.O. Box 513, 5600 MB Eindhoven, The Netherlands; part of this work was done while MM was at Stanford University, Stanford, CA 94305, US (mmandjes@science . uva.nl). 


\section{Introduction}

Single-server queues in which the server takes vacations naturally arise as models for a wide range of computer-, communication- and production systems. The vacation times may, e.g., represent server breakdowns, or periods in which the server processes work generated by another class of customers. We refer to [4,5] for surveys on vacation queues, and to [10] for an extensive textbook treatment.

It is almost invariably assumed that the length of a vacation is independent of the length of the previous busy period. This may be highly unrealistic: in many systems of practical interest, these quantities will be correlated. To gain insight into the impact of these dependencies, we have considered in [3] a vacation queue in which the length of a vacation is positively correlated with the length of the preceding busy period. Another extension in [3] with respect to classical vacation queues was the assumption that the buffer content or storage level during both the busy periods and the vacation periods evolves according to Lévy processes $[1,7,8,9]$; the 'traditional literature' predominantly focuses on a subclass of Lévy processes, viz., compound Poisson input (leading to M/G/1-type of queueing models). It is noted that for Lévy processes a rich body of powerful results has been developed, on which we extensively relied in [3].

In [2] another form of correlation between busy periods and vacations is considered, yielding relatively explicit expressions for the stationary distribution of the buffer content, both at beginnings of busy periods and in continuous time. The present paper can be regarded as the companion paper of [2]. In both papers, once more, the buffer content or storage level during both the busy periods and the vacation periods evolves according to Lévy processes.

In the present paper we consider a model that may give rise to both positive and negative correlations between the length of a busy period and the subsequent vacation. More specifically, if the length of the busy period is $\tau$, then the length of the subsequent vacation is $c \tau$ if $\tau<T_{\lambda}$, where $T_{\lambda}$ is an independent, exponentially distributed clock time. Else, the queue remains idle for some exponentially distributed period (with mean $\delta^{-1} ; \delta$ can be chosen $\infty$, corresponding to the case of no idle time), and the next busy period is assumed to start at some random level, distributed according to the random variable $B$.

We analyze the steady-state workload of this Lévy-driven storage system, both at beginnings of busy periods and in continuous time. An interesting feature of the model under consideration is that it allows for infinitely many busy-vacation cycles in a finite time interval, making the analysis non-standard. Precise conditions can be derived under which such an 'explosion' may occur; in case explosion occurs with probability 0 , we can derive the Laplace-Stieltjes transform of the stationary distribution of the buffer content at beginnings of busy periods, whereas in case explosion occurs with positive probability such a stationary distribution does not exist. Irrespective of whether the storage process, embedded at beginnings of busy periods, has a stationary distribution, the steady-state workload of the continuous-time storage process can be determined, though. 
The paper is organized as follows. Section 2 contains a detailed model description, as well as preliminary results on Lévy processes and on the storage level at the embedded points of busy period beginnings. Section 3 presents a number of auxiliary results. In Section 4 we distinguish between various types of Lévy processes, giving rise to different recurrence properties. We identify conditions under which the storage process embedded at busy period beginnings is irreducible and Harris positive recurrent, as well as conditions under which the continuous-time storage process has a proper stationary distribution. In Section 5 we determine the Laplace-Stieltjes transform of the steady-state embedded process (given it exists), as well as the continuous-time storage process. We conclude the paper in Section 6 with an analysis of the distribution of the regeneration time, i.e., the time between two successive epochs in which the process starts from an independent initial position distributed like the random variable $B$.

\section{Model description and some preliminaries}

In this section we first introduce the model, as well as the notation needed later on in the paper. We then present a number of preliminary computations which seem to lead to the steady-state distribution in a fairly straightforward way. Then we point out that this is not quite true, due to the model's non-trivial behavior; more precisely, with positive probability an infinite number of cycles occurs in a finite time interval. We conclude this section with some reflections on the dependence between the durations of the vacations and the preceding busy periods.

Model. Consider a storage process which evolves as follows. At time zero the process has an arbitrary initial position $X(0)$.

$\mathbf{I}^{\mathrm{a}}$ If $X(0)=0$, then start from an independent initial position distributed like a positive random variable $B$ with Laplace-Stieltjes transform (LST) $\beta(\alpha)=\mathbb{E} e^{-\alpha B}$, from which the process restarts in a manner that will be described in Step $\mathbf{I}^{\mathrm{b}}$.

I $^{\mathrm{b}}$ If $X(0)>0$, in particular when $X(0) \sim B$, then the storage process behaves after 0 like a Lévy process $X=\{X(t) \mid t \geq 0\}$ with no negative jumps, in the sense that $\bar{X}:=\{X(t)-X(0) \mid t \geq 0\}$ is a Lévy process (that is independent of the value of $X(0))$. We assume that $\bar{X}$ has the Lévy exponent

$$
\varphi(\alpha)=\log \mathbb{E} e^{-\alpha \bar{X}(1)}=\mu \alpha+\frac{\sigma^{2} \alpha^{2}}{2}+\int_{(0, \infty)}\left(e^{-\alpha x}-1+\alpha x 1_{\{0<x \leq 1\}}\right) \nu(\mathrm{d} x),
$$

where $\nu$ is its Lévy measure, satisfying a negative-drift condition

$$
-\varphi^{\prime}(0)=-\mu+\int_{(1, \infty)} x \nu(\mathrm{d} x)=\mathbb{E} \bar{X}(1)<0 .
$$


It is well known that $X$ (and $\bar{X}$ ) is of unbounded variation if either $\int_{(0,1]} x \nu(\mathrm{d} x)=\infty$ or $\sigma^{2}>0$ [7, Section 2.6.1]; we write $X \in \mathscr{U}$. Otherwise $X$ is of bounded variation (which we denote in the sequel by $X \in \mathscr{B})$, and then $X(t)$ can be written as $X(0)+J(t)-b t$, where $J$ is a pure jump subordinator with $J(0)=0$ and $b:=\mu+\int_{(0,1]} x \nu(\mathrm{d} x)[7$, Lemma 2.13]. In the latter case (1) and (2) read

$$
\varphi(\alpha)=b \alpha-\int_{(0, \infty)}\left(1-e^{-\alpha x}\right) \nu(\mathrm{d} x) \quad \text { and } \quad \varphi^{\prime}(0)=b-\int_{(0, \infty)} x \nu(\mathrm{d} x) .
$$

Now introduce the hitting time of level 0: $\tau:=\inf \{t>0 \mid X(t)=0\}$. In the remainder of the paper we denote $\psi(s):=\inf \{\alpha \mid \varphi(\alpha)>s\}$ (to be thought of as the inverse of the Lévy exponent $\varphi(\cdot))$. Denoting by $\mathbb{E}_{z}(\cdot)$ the expected value operator when $X(0)=z$ and $\mathbb{P}_{z}(\cdot)$ the associated probability measure, it is easily seen that, due to the absence of negative jumps, for $s \geq 0, \mathbb{E}_{z} e^{-s \tau}=e^{-\gamma_{s} z}$; a straightforward martingale argument shows that in fact $\gamma_{s}=\psi(s)$ [7, Thm. 3.12]. In particular, under the stated conditions it is well known that $\varphi(\cdot)$ is an increasing convex function with $\varphi(0)=0$ and $\lim _{\alpha \rightarrow \infty} \varphi(\alpha)=\infty$, so that $\psi(\cdot)$ is well defined on $[0, \infty)$, increasing and concave with $\psi(0)=0$ and $\psi^{\prime}(0)=1 / \varphi^{\prime}(0)<\infty$. In particular $\mathbb{E}_{z} \tau=\psi^{\prime}(0) z$ so that $\tau_{1}$ is $\mathbb{P}_{z}$-almost surely finite for any $z \geq 0$.

We let the storage process behave as the process $X$ until $\tau$. Go to Step II.

II We let $T_{\lambda} \sim \exp (\lambda)$ be an independent exponential clock. If $\tau \geq T_{\lambda}$, then we wait some exponential time with mean $\delta^{-1}$ and go to Step $\mathbf{I}^{\mathrm{a}}$. If, on the contrary, $\tau<T_{\lambda}$, that is, if $\tau$ is sufficiently small, then starting off from level 0 at time $\tau$ the storage process will evolve, for a period $c \tau$ for some $c>0$, according to some nondecreasing Lévy process (i.e., subordinator) $Y$. This Lévy process is uniquely characterized through its Lévy exponent; with $\nu_{Y}$ denoting the corresponding Lévy measure, and $a \geq 0$,

$$
\eta(\alpha)=-\log \mathbb{E} e^{-\alpha Y(1)}=a \alpha+\int_{(0, \infty)}\left(1-e^{-\alpha x}\right) \nu_{Y}(\mathrm{~d} x),
$$

$\eta(\alpha)$ being the negative of its Lévy exponent. We assume that

$$
\mathbb{E} Y(1)=a+\int_{(0, \infty)} x \nu_{Y}(\mathrm{~d} x)<\infty .
$$

If at time $\tau(1+c)$ the storage level is 0 , then we wait some exponential time with mean $\delta^{-1}$ and go to Step $\mathbf{I}^{\mathrm{a}}$; else, we go to Step $\mathbf{I}^{\mathrm{b}}$, that is, we revert to the original Lévy process $X$, but now with initial state $Y(c \tau)$.

A sequence of Step $\mathbf{I}^{\mathrm{a} / \mathrm{b}}$ and Step $\mathbf{I I}$ is called a cycle; we could call Step $\mathbf{I}^{\mathrm{b}}$ a busy period of the queue (as the storage level on average decreases, due to $\varphi^{\prime}(0)>0$ ), whereas Step II can be thought of as a vacation (as the storage level increases). According to the above description, the storage process alternates between Steps $\mathbf{I}^{\mathrm{a} / \mathrm{b}}$ and $\mathbf{I I}$. The buffer dynamics are illustrated in Fig. 1. 

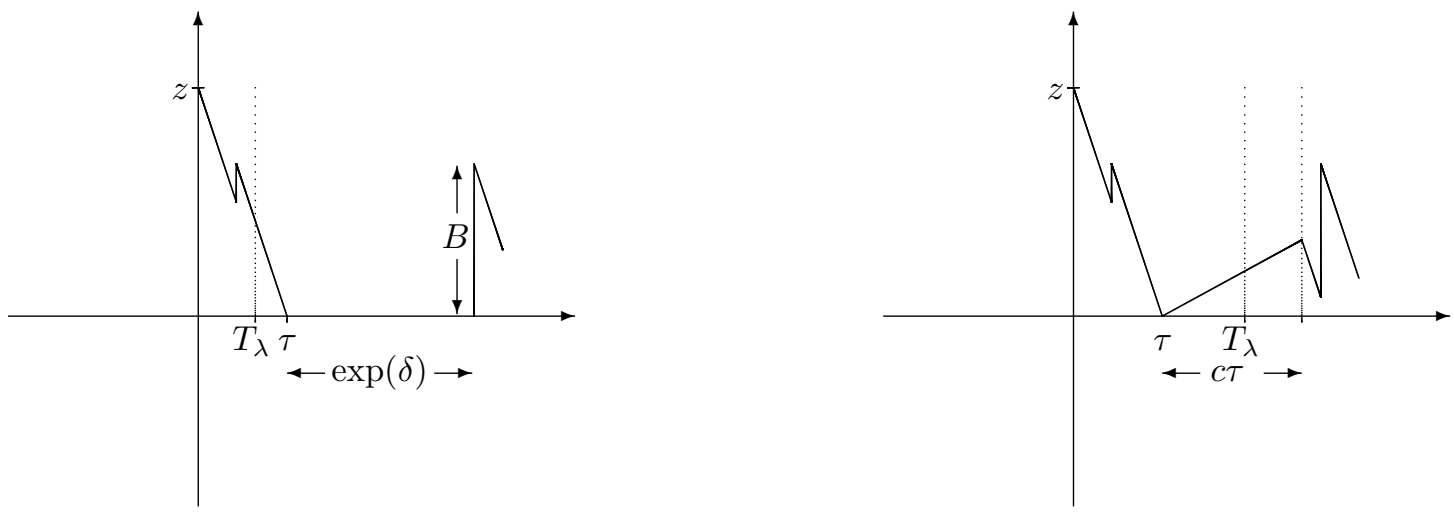

Figure 1: Illustration of buffer dynamics, for the case in which $X$ is compound Poisson, and $Y$ is a linear drift. Left: $T_{\lambda}<\tau$, right: $T_{\lambda} \geq \tau$.

Remark 1 Notice that cycles are not i.i.d. (as they do not start at the same initial level). Regeneration points are epochs at which the storage level is 0 at the end of a cycle, which can be due to (i) $\tau \geq T_{\lambda}$, (ii) $\tau<T_{\lambda}$, but $Y(c \tau)=0$. In other words: regeneration periods start with Step $\mathbf{I}^{\mathrm{a}}$.

Preliminary computations, and additional notation. Let $Z_{n}$ be the storge level after $n$ cycles; clearly $\left\{Z_{n} \mid n \in \mathbb{N}\right\}$ is Markovian. To get a handle on the steady-state distribution of the $Z_{n}$, it is useful to compute the LST of the storage level after one cycle, for an arbitrary initial position $z$. It is elementary that for $z>0$,

$$
\mathbb{P}_{z}\left(\tau \geq T_{\lambda}\right)=\mathbb{E}_{z} \mathbb{P}_{z}\left(\tau \geq T_{\lambda} \mid \tau\right)=\mathbb{E}_{z}\left(1-e^{-\lambda \tau}\right)=1-e^{-\psi(\lambda) z}
$$

In a similar fashion, with $h(\alpha):=\psi(\lambda+c \eta(\alpha))$,

$$
\begin{aligned}
\mathbb{E}_{z} e^{-\alpha Y(c \tau)} 1_{\left\{\tau<T_{\lambda}\right\}} & =\mathbb{E}_{z} \mathbb{E}_{z}\left[e^{-\alpha Y(c \tau)} 1_{\left\{\tau<T_{\lambda}\right\}} \mid \tau\right]=\mathbb{E}_{z} \mathbb{E}_{z}\left[e^{-\alpha Y(c \tau)} \mid \tau\right] \mathbb{P}_{z}\left(\tau<T_{\lambda} \mid \tau\right) \\
& =\mathbb{E}_{z} e^{-\eta(\alpha) c \tau} e^{-\lambda \tau}=\mathbb{E}_{z} e^{-(\lambda+c \eta(\alpha)) \tau}=e^{-h(\alpha) z}
\end{aligned}
$$

Hence, for $z>0$,

$$
\mathbb{E}_{z} e^{-\alpha Z_{1}}=e^{-h(\alpha) z}+1-e^{-h(0) z} .
$$

When $X(0)=0$ we have by the above description that (5) implies that

$$
\mathbb{E}_{0} e^{-\alpha Z_{1}}=\mathbb{E}_{B} e^{-\alpha Z_{1}}=\beta(h(\alpha))+1-\beta(h(0)) .
$$

Combining (5) and (6), we obtain that for all $z \geq 0$,

$$
\mathbb{E}_{z} e^{-\alpha Z_{1}}=1-\left[e^{-h(0) z}-e^{-h(\alpha) z}\right]-[\beta(h(0))-\beta(h(\alpha))] 1_{\{z=0\}} .
$$


With $Z$ being a random variable with as distribution the steady-state distribution of the $Z_{n}$, we observe that the previous equation entails that $F(\alpha):=\mathbb{E} e^{-\alpha Z}$ obeys

$$
F(\alpha)=1-F(h(0))+F(h(\alpha))+(\beta(h(\alpha))-\beta(h(0))) \pi_{0},
$$

where $\pi_{0}:=\mathbb{P}(Z=0)$, if the steady-state distribution of the $Z_{n}$ indeed exists.

From (4), if either $a>0$ or $\nu_{Y}(0, \infty)=\infty$, then $\lim _{\alpha \rightarrow \infty} \eta(\alpha)=\infty$. Otherwise, when $a=0$ and $\lambda_{Y}:=\nu_{Y}(0, \infty)<\infty$, then $\lim _{\alpha \rightarrow \infty} \eta(\alpha)=\lambda_{Y}$. This implies that if $Y$ is not a 'driftless' compound Poisson process (that is, a pure jump compound Poisson process), then, for any $t \geq 0$, it holds that $Y(t)>0$ almost surely:

$$
\mathbb{P}(Y(t)=0)=\lim _{\alpha \rightarrow \infty} \mathbb{E} e^{-\alpha Y(t)}=\lim _{\alpha \rightarrow \infty} e^{-\eta(\alpha) t}=0 ;
$$

when, on the contrary, $Y$ is a pure jump compound Poisson process (which we denote by $Y \in \mathscr{C}), \mathbb{P}(Y(t)=0)=e^{-\lambda_{Y} t}$, as expected from the fact that the time until the first jump is exponentially distributed with rate $\lambda_{Y}$.

For later reference, we here also introduce $\tau_{n}$ as the time from the end of the $n$th cycle until the content first reaches zero. Denote by $T_{\lambda, n}$ the exponential time which is compared with $\tau_{n}$ in cycle $n$, and assume that $\left\{T_{\lambda, n} \mid n \in \mathbb{N}\right\}$ are i.i.d $\exp (\lambda)$ distributed random variables, which are in addition independent of the processes $X$ and $Y$.

A paradox? Later, we will show (see Lemma 1) that under certain conditions there is a unique $0<\alpha^{\star}<\infty$ such that $h\left(\alpha^{\star}\right)=\alpha^{\star}$, and $h(\infty)=\infty$. Let us assume for the moment that these conditions are met. Now, one idea for trying to analyze our model is to first find a stationary distribution for the embedded Markov process $\left\{Z_{n} \mid n \in \mathbb{N}\right\}$ and then use techniques from point process theory and martingale analysis to relate the stationary distribution of the continuous time process to that of this embedded Markov chain, cf. [2, 3].

We now wish to point out a problem regarding the existence of the stationary distribution of the embedded Markov chain. Let $B$ be such that $\beta(\infty)=0$. If there is such a stationary distribution $Z$ then on the one hand its LST $F(\alpha)=\mathbb{E} e^{-\alpha Z}$ is of the form $\pi_{0}+\left(1-\pi_{0}\right) \zeta(\alpha)$, with $\pi_{0}=\mathbb{P}(Z=0)$; here $\zeta(\alpha)$ is the LST of a strictly positive random variable (so that $\zeta(\infty)=0)$. On the other hand, (5) and (6) imply that it is also of the form

$$
\pi_{0}(\beta(h(\alpha))+1-\beta(h(0)))+\left(1-\pi_{0}\right)(\zeta(h(\alpha))+1-\zeta(h(0))) .
$$

Letting $\alpha \rightarrow \infty$ in (8) we have that

$$
\pi_{0}=\pi_{0}(1-\beta(h(0)))+\left(1-\pi_{0}\right)(1-\zeta(h(0))) .
$$

This leads to the equation

$$
\left(1-\pi_{0}\right) \zeta(\alpha)=\pi_{0} \beta(h(\alpha))+\left(1-\pi_{0}\right) \zeta(h(\alpha)) .
$$

We can now substitute the fixed point $\alpha^{\star}$ in this equation. But this leads to the (clearly incorrect) conclusion that $\pi_{0} \beta\left(\alpha^{\star}\right)$ is zero! 
Did we make an error in this calculation? The answer is no. The error was in the assumption that a stationary distribution for $\left\{Z_{n} \mid n \in \mathbb{N}\right\}$ exists in this case. We will show that explosion may occur, in that there may be (with positive probability) an infinite number of cycles within a finite time interval. More precisely: we conclude from the above that in situations in which there is a $\alpha^{\star}$ such that $h\left(\alpha^{\star}\right)=\alpha^{\star}$, and in addition $h(\infty)=\infty$ and $\beta(\infty)=0$, the process $\left\{Z_{n} \mid n \in \mathbb{N}\right\}$ cannot have a proper stationary distribution.

The interesting fact is that our process will turn out to be regenerative where the probability of having an infinite number of on/off cycles is strictly between zero and one. A demonstration of this phenomenon in a very simple case is given in Example 1 that concludes Section 4, for the case of deterministic $X(\cdot)$ and $Y(\cdot)$ processes and $0<c<1$. In this case, starting with some value $z$, an infinite succession of busy and idle cycles, with geometrically decreasing lengths, may occur in a regenerative cycle. There is a positive probability that all busy periods are smaller than $T_{\lambda}$, but, fortunately, we can analyze the stationary distribution of the continous-time storage model regardless of whether this phenomenon can happen or not, as demonstrated in Section 5.

Correlation between vacations and the preceding active period. Clearly, the model presented in this section can be regarded as a queue with server vacations. During times in which the storage level evolves according to the Lévy process $X$, one could say that the server is active, while during times in which it evolves according to $Y$, there is a server vacation. As explained above, only if the exponential clock (with parameter $\lambda$ ) expires after the end of the busy period (i.e., the period in which the server is active), there is a genuine vacation (as otherwise, the queue remains empty for an exponentially distributed time with mean $\delta^{-1}$, and then a service requirement $B$ is generated). Clearly, these system mechanics lead to a storage process in which the vacation durations heavily depend on the length of the previous busy period, thus departing from the 'classical literature' where these were commonly assumed to be independent.

In many situations of practical interest, there is a positive correlation between vacations and the lengths of the previous busy periods [3]. For example, in polling models a relatively long visit of the server to a tagged queue probably leads to a substantial accumulation of work in subsequent queues, and hence to a relatively long intervisit time of the tagged queue. In other applications more involved correlation structures may arise.

The idea of the model discussed in the present paper is that when the busy period is sufficiently small $\left(\tau<T_{\lambda}\right)$ then there is an interruption of size $c \tau$, and when it is relatively large then there is no interruption (that is, the system remains empty for an exponentially distributed time with mean $\delta^{-1}$ ).

To gain some insight into the correlation structure it leads to, we compute the covariance between the length of the busy period $\tau$ and the length of the interruption $c \tau 1_{\left\{\tau<T_{\lambda}\right\}}$. From $\mathbb{E}_{z} \tau=z \psi^{\prime}(0)$,

$$
\mathbb{E}_{z}\left[c \tau 1_{\left\{\tau<T_{\lambda}\right\}}\right]=c \cdot \mathbb{E}_{z} \tau e^{-\lambda \tau}=-c \cdot \frac{\mathrm{d}}{\mathrm{d} \lambda} \mathbb{E}_{z} e^{-\lambda \tau}=-c \cdot \frac{\mathrm{d}}{\mathrm{d} \lambda} e^{-\psi(\lambda) z}=c \psi^{\prime}(\lambda) z e^{-\psi(\lambda) z},
$$


and

$$
\begin{aligned}
\mathbb{E}_{z}\left[c \tau^{2} 1_{\left\{\tau<T_{\lambda}\right\}}\right] & =c \cdot \mathbb{E}_{z} \tau^{2} e^{-\lambda \tau}=c \cdot \frac{\mathrm{d}^{2}}{\mathrm{~d} \lambda^{2}} \mathbb{E}_{z} e^{-\lambda \tau} \\
& =c \cdot \frac{\mathrm{d}^{2}}{\mathrm{~d} \lambda^{2}} e^{-\psi(\lambda) z}=c e^{-\psi(\lambda) z}\left(\left(\psi^{\prime}(\lambda)\right)^{2} z^{2}-\psi^{\prime \prime}(\lambda) z\right),
\end{aligned}
$$

we see that the covariance between the busy period and the interruption period is given by

$$
c z e^{-\psi(\lambda) z}\left[\left(\psi^{\prime}(\lambda)-\psi^{\prime}(0)\right) \psi^{\prime}(\lambda) z-\psi^{\prime \prime}(\lambda)\right] .
$$

Since $\psi$ is concave, then either it is linear and then $\psi^{\prime}(\lambda)=\psi^{\prime}(0)$ as well as $\psi^{\prime \prime}(\lambda)=0$ or it is strictly concave, in which case $\psi^{\prime}(\lambda)<\psi^{\prime}(0)$ and $\psi^{\prime \prime}(\lambda)<0$. In this case when

$$
z>\frac{-\psi^{\prime \prime}(\lambda)}{\psi^{\prime}(\lambda)\left(\psi^{\prime}(0)-\psi^{\prime}(\lambda)\right)}
$$

then the covariance is negative, but otherwise it is not. To make a more general claim on the (sign of the) covariance, we should weigh Expression (9) (i.e., the covariance for initial storage level $z$ ) by the distribution of $Z$ (that is, the limiting distribution of the $Z_{n}$ ), if this distribution exists; the distribution of $Z$ will be discussed in Section 5 .

\section{Auxiliary results}

In this section we prove a number of auxiliary results that will be used in Section 4 when the recurrence of $\left\{Z_{n} \mid n \in \mathbb{N}\right\}$ is studied. We first analyze in Lemma 1 the behavior of $h^{n}(\alpha)$ for $n \rightarrow \infty$; it turns out that in our analysis an important role is played by conditions under which there is a unique fixed point $\alpha^{\star}$ (solving $\alpha=h(\alpha)$, for a function $h(\cdot)$ specified below). In Lemma 3, relying on the technical result stated in Lemma 2, we determine the distribution of the number of cycles $N$ in a regeneration period. The result entails that the specific properties of the Lévy processes $X$ and $Y$ essentially determine whether $N$ is defective or not.

Definition 1 We denote by $X \in \mathscr{B}_{+}$that $X \in \mathscr{B}$ with $b>c a$, and by $X \in \mathscr{B}_{-}$that $X \in \mathscr{B}$ with $b \leq c a$; see (3) for the definition of $b$.

Lemma 1 Let $h(\alpha):=\psi(c \eta(\alpha)+\lambda), h^{0}(\alpha):=\alpha$ and $h^{n}(\alpha):=h\left(h^{n-1}(\alpha)\right)$ for $n=1,2, \ldots$..

(i) If either $X \in \mathscr{U}$, or $X \in \mathscr{B}_{+}$, then there is a unique fixed point $0<\alpha^{\star}<\infty$ for which $h\left(\alpha^{\star}\right)=\alpha^{\star}$. In this case $h^{n}(\alpha) \rightarrow \alpha^{\star}$ for every $\alpha \geq 0$ and $h^{\prime}\left(\alpha^{\star}\right)<1$. Moreover, for every $0 \leq \alpha_{2}<\alpha_{1} \leq \infty$ and $m \in\{1, \ldots, n\}, n=1,2, \ldots$,

$$
0 \leq h^{n}\left(\alpha_{1}\right)-h^{n}\left(\alpha_{2}\right) \leq h^{\prime}\left(h^{m}(0)\right)^{n-m}\left(h^{m}\left(\alpha_{1}\right)-h^{m}\left(\alpha_{2}\right)\right) .
$$

(ii) If $X \in \mathscr{B}_{-}$, then $h^{n}(\alpha) \rightarrow \infty$ as $n \rightarrow \infty$ for each $\alpha \geq 0$. In this case $\lim _{\alpha \rightarrow \infty} h^{\prime}(\alpha)=c a / b$ and $h^{n}(0) \geq n \psi(\lambda)$ for $n=0,1, \ldots$. 
Proof: We first prove that a unique fixed point $\alpha^{\star}$ exists under the conditions of case (i). Since $\psi$ and $\eta$ are both nondecreasing and concave, so is $h(\alpha)$. Hence, $h(\alpha)-\alpha$ is concave and consequently also continuous. Since $h(0)=\psi(\lambda)>0$ there is a unique fixed point if and only if for some $\alpha>0, h(\alpha)<\alpha$. As $\psi$ is increasing, this is equivalent to requiring that, for some $\alpha>0, c \eta(\alpha)+\lambda<\varphi(\alpha)$ or $\varphi(\alpha)-c \eta(\alpha)>\lambda$. Since $\varphi(\alpha)-c \eta(\alpha)$ is convex, a necessary and sufficient condition is that

$$
\lim _{\alpha \rightarrow \infty}\left(\varphi^{\prime}(\alpha)-c \eta^{\prime}(\alpha)\right)>0 .
$$

Noting that

$$
\varphi^{\prime}(\alpha)=\mu+\sigma^{2} \alpha+\int_{(0,1]}\left(1-e^{-\alpha x}\right) x \nu(\mathrm{d} x)-\int_{(1, \infty)} e^{-\alpha x} x \nu(\mathrm{d} x),
$$

it follows that if $X \in \mathscr{U}$ (i.e., either $\sigma^{2}>0$ or $\int_{(0,1]} x \nu(\mathrm{d} x)=\infty$ ), then $\varphi^{\prime}(\alpha) \rightarrow \infty$ as $\alpha \rightarrow \infty$. Suppose now that $X \in \mathscr{B}$, in which case

$$
\varphi^{\prime}(\alpha) \rightarrow \mu+\int_{(0,1]} x \nu(\mathrm{d} x)=b
$$

as $\alpha \rightarrow \infty$. Furthermore,

$$
\eta^{\prime}(\alpha)=a+\int_{(0, \infty)} e^{-\alpha x} x \nu_{Y}(\mathrm{~d} x) \rightarrow a
$$

as $\alpha \rightarrow \infty$. Conclude that $\varphi^{\prime}(\alpha)-c \eta^{\prime}(\alpha) \rightarrow b-c a$, which is positive provided that $b>c a$. We have now proven that under the stated conditions there is indeed a unique fixed point $\alpha^{\star}$.

We now consider the behavior of $h^{n}(\alpha)$ as $n \rightarrow \infty$. It is clear that $\alpha^{\star}>0$ and, as $h$ is increasing, $0<\psi(\lambda)=h(0)<h\left(\alpha^{\star}\right)=\alpha^{\star}$ and thus $h^{n-1}(0)<h^{n}(0)<h^{n-1}\left(\alpha^{\star}\right)=\alpha^{\star}$. Therefore $h^{n}(0)$ is a bounded monotone sequence, and consequently has a limit. Since $h$ is continuous and $h^{n}(0)=h\left(h^{n-1}(0)\right)$, the limit must be the unique fixed point $\alpha^{\star}$. For $0<\alpha<\alpha^{\star}$ we have that $h^{n}(0)<h^{n}(\alpha)<h^{n}\left(\alpha^{\star}\right)=\alpha^{\star}$, so that $h^{n}(\alpha) \rightarrow \alpha^{\star}$ for every $0 \leq \alpha \leq \alpha^{\star}$. Now concentrate on $\alpha \geq \alpha^{\star}$. Since $h(\alpha)-\alpha$ is concave, $h(0)-0=\psi(\lambda)>0$ and $h\left(\alpha^{\star}\right)-\alpha^{\star}=0$ it follows that on $\left[\alpha^{\star}, \infty\right)$ the function $h(\alpha)-\alpha$ is decreasing. In particular, for $\alpha>\alpha^{\star}, h(\alpha)-\alpha<0$, so that $\alpha^{\star}=h\left(\alpha^{\star}\right)<h(\alpha)<\alpha$, and hence $\alpha^{\star}<h^{n}(\alpha)<h^{n-1}(\alpha)$. Once again, by monotonicity and continuity, $h^{n}(\alpha)$ converges to the unique fixed point $\alpha^{\star}$. Since $h(0)-0>0, h\left(\alpha^{\star}\right)-\alpha^{\star}=0$ and the fact that $h(\alpha)-\alpha$ is concave, it follows that necessarily it is decreasing at $\alpha^{\star}$ and thus $h^{\prime}\left(\alpha^{\star}\right)-1<0$.

Equation (10) follows from

$$
h^{n}\left(\alpha_{1}\right)-h^{n}\left(\alpha_{2}\right)=h^{\prime}(u)\left(h^{n-1}\left(\alpha_{1}\right)-h^{n-1}\left(\alpha_{2}\right)\right)
$$

for some $h^{n-1}\left(\alpha_{2}\right) \leq u \leq h^{n-1}\left(\alpha_{1}\right)$. Since $h^{\prime}$ is nonincreasing and $h^{n-1}\left(\alpha_{2}\right) \geq h^{n-1}(0) \geq$ $h^{m}(0)$, we have from (11) that $h^{\prime}(u) \leq h^{\prime}\left(h^{m}(0)\right)$ and

$$
h^{n}\left(\alpha_{1}\right)-h^{n}\left(\alpha_{2}\right) \leq h^{\prime}\left(h^{m}(0)\right)\left(h^{n-1}\left(\alpha_{1}\right)-h^{n-1}\left(\alpha_{2}\right)\right),
$$


and we can proceed by induction. This concludes the proof of claim (i).

We now proceed with the proof of claim (ii), featuring the situation that $X \in \mathscr{B}_{-}$. In this case, as $\varphi^{\prime}(\alpha)-c \eta^{\prime}(\alpha)$ is nondecreasing with a nonpositive limit (use $b \leq c a$ ), it follows that it is nonpositive for all $\alpha \geq 0$. Hence, $\varphi(\alpha)-c \eta(\alpha)$ is nonincreasing and thus nonpositive for all $\alpha \geq 0$, and hence no solution to $\varphi(\alpha)-c \eta(\alpha)=\lambda$ exists. This implies that in this case no finite solution to $h(\alpha)=\alpha$ exists either. If we show that $h^{n}(0) \rightarrow \infty$ as $n \rightarrow \infty$, then we have also proven that $h^{n}(\alpha) \rightarrow \infty$, due to the fact that $h^{n}(0) \leq h^{n}(\alpha)$ for each $\alpha \geq 0$. Now, $0<\psi(\lambda)=h(0)$, hence by induction $h^{n-1}(0) \leq h^{n}(0)$ so that $\lim _{n \rightarrow \infty} h^{n}(0)$ exists and is either finite or infinite. If it is finite, then by continuity of $h$ this limit must be a fixed point. Since no such fixed point exists, it has to be infinite.

When $X \in \mathscr{B}_{-}$, then, due to $\varphi^{\prime}(0)>0$, it follows that necessarily $b>0$ and hence $a>0$ as well. Thus, $Y$ cannot be a compound Poisson process and we recall that in this situation $\eta(\alpha) \rightarrow \infty$ as $\alpha \rightarrow \infty$. Therefore, $\psi(\lambda+c \eta(\alpha)) \rightarrow \infty$ as $\alpha \rightarrow \infty$ and we have that

as $\alpha \rightarrow \infty$.

$$
h^{\prime}(\alpha)=\psi^{\prime}(\lambda+c \eta(\alpha)) c \eta^{\prime}(\alpha)=\frac{c \eta^{\prime}(\alpha)}{\varphi^{\prime}(\psi(\lambda+c \eta(\alpha)))} \rightarrow \frac{c a}{b}
$$

Finally, $h^{n+1}(0)-h^{n}(0)=h^{\prime}\left(\beta_{n}\right)\left(h^{n}(0)-h^{n-1}(0)\right)$ for some $h^{n-1}(0) \leq \beta_{n} \leq h^{n}(0)$. Since $h^{\prime}$ is nonincreasing ( $h$ is concave) then $h^{\prime}\left(\beta_{n}\right) \geq c a / b \geq 1$, so that by induction $h^{n+1}(0)-h^{n}(0) \geq$ $h(0)=\psi(\lambda)$ for $n \geq 1$ and clearly also for $n=0$. Thus, upon summation, we have that $h^{n}(0) \geq n \psi(\lambda)$.

Remark 2 We note that if $Y$ is a pure jump subordinator, that is, if $a=0$, then the existence of a unique fixed point is assured regardless of whether or not $X$ is of unbounded variation. The only condition needed then is that $\varphi^{\prime}(0)$ be positive.

Recall that regeneration points are epochs at which the storage level is 0 at the end of a cycle, which can be due to (i) $\tau_{n} \geq T_{\lambda, n}$ (for some $n$ ), (ii) $\tau_{n}<T_{\lambda, n}$, but $Y\left(c \tau_{n}\right)=0$ (for some $n$ ). We now study the number of cycles until the system regenerates. To this end, denote

$$
N:=\inf \left\{n \in\{1,2, \ldots\} \mid \tau_{n} \geq T_{\lambda, n} \text { or } Z_{n}=0\right\} 1_{\left\{Z_{0}>0\right\}},
$$

so that $\{N>0\}=\left\{Z_{0}>0\right\}$ and for $n=1,2, \ldots$,

$$
\{N>n\}=\left\{Z_{0}>0, \ldots, Z_{n}>0, \tau_{1}<T_{\lambda, 1}, \ldots, \tau_{n}<T_{\lambda, n}\right\} .
$$

The following lemma will appear useful when establishing a number of structural properties of the random variable $N$.

Lemma 2 For some nonnegative measurable trivariate function $u$, denote for $n=0,1, \ldots$,

$$
u_{n}(z):=\mathbb{E}_{z} u\left(Z_{n}, \tau_{n+1}, Z_{n+1}\right) 1_{\{N>n\}},
$$

so that $u_{0}(z)=1_{\{z>0\}} \mathbb{E}_{z} u\left(z, \tau_{1}, Z_{1}\right)$ and $u_{n}(0)=0$ for $n=1,2, \ldots$. Then

$$
u_{n}(z)=\mathbb{E}_{z}\left[e^{-\lambda \tau_{1}} \mathbb{E}_{z}\left[u_{n-1}\left(Y\left(c \tau_{1}\right)\right) \mid \tau_{1}\right]\right]
$$

for $n=1,2, \ldots$ 
Proof: The proof is a straightforward computation. For $n=1,2, \ldots$,

$$
\begin{aligned}
u_{n}(z) & =\mathbb{E}_{z}\left[u\left(Z_{n}, \tau_{n+1}, Z_{n+1}\right) 1_{\{N>n\}}\right] \\
& =\mathbb{E}_{z}\left[1_{\left\{\tau_{1}<T_{\lambda, 1}\right\}} \mathbb{E}_{z}\left[u\left(Z_{n}, \tau_{n+1}, Z_{n+1}\right) 1_{\{N>n\}} \mid \tau_{1}, T_{\lambda, 1}, Y\left(c \tau_{1}\right)\right]\right],
\end{aligned}
$$

where it is used that under $\{N>n\}$ we know that $\tau_{1}<T_{\lambda, 1}$. But then we can express the latter expression in terms of $u_{n}(\cdot)$; to this end realize that

$$
\begin{aligned}
& \mathbb{E}_{z}\left[u\left(Z_{n}, \tau_{n+1}, Z_{n+1}\right) 1_{\{N>n\}} \mid \tau_{1}, T_{\lambda, 1}, Y\left(c \tau_{1}\right)\right] \\
& \quad=\mathbb{E}_{Y\left(c \tau_{1}\right)} u\left(Z_{n-1}, \tau_{n}, Z_{n}\right) 1_{\{N>n-1\}}=u_{n-1}\left(Y\left(c \tau_{1}\right)\right) .
\end{aligned}
$$

This means that (12) further reduces to

$$
\begin{aligned}
& \mathbb{E}_{z}\left[1_{\left\{\tau_{1}<T_{\lambda, 1}\right\}} u_{n-1}\left(Y\left(c \tau_{1}\right)\right)\right]=\mathbb{E}_{z}\left[\mathbb{E}_{z}\left[1_{\left\{\tau_{1}<T_{\lambda, 1}\right\}} u_{n-1}\left(Y\left(c \tau_{1}\right)\right) \mid \tau_{1}\right]\right] \\
& \quad=\mathbb{E}_{z}\left[\mathbb{E}_{z}\left[1_{\left\{\tau_{1}<T_{\lambda, 1}\right\}} \mid \tau_{1}\right] \cdot \mathbb{E}_{z}\left[u_{n-1}\left(Y\left(c \tau_{1}\right)\right) \mid \tau_{1}\right]\right]=\mathbb{E}_{z}\left[e^{-\lambda \tau_{1}} \mathbb{E}_{z}\left[u_{n-1}\left(Y\left(c \tau_{1}\right)\right) \mid \tau_{1}\right]\right] .
\end{aligned}
$$

This proves the claim.

Recall that if $Y \in \mathscr{C}$ (that is, $Y$ is compound Poisson), then $\lambda_{Y}:=\nu_{Y}(0, \infty)=\lim _{\alpha \rightarrow \infty} \eta(\alpha)<$ $\infty$, and otherwise it is infinite. Thus, when $Y \in \mathscr{C}$, we denote $h(\infty)=\psi\left(\lambda+c \lambda_{Y}\right)$; when $Y \notin \mathscr{C}$, then $h(\infty)=\infty$ and consequently $h^{n}(\infty)=\infty$ for all $n=1,2, \ldots$

The following lemma determines the distribution of the number of cycles $N$ per regeneration period. It particularly specifies under which conditions $N$ is $\mathbb{P}_{z}$-defective.

Lemma 3 For each $z>0$ and $n=1,2, \ldots$,

$$
\mathbb{P}_{z}(N>n)= \begin{cases}e^{-h^{n}(0) z}-e^{-h^{n}(\infty) z} & \text { if } Y \in \mathscr{C} \\ e^{-h^{n}(0) z} & \text { otherwise. }\end{cases}
$$

Consequently, if neither (i) $Y \in \mathscr{C}$, nor (ii) $X \in \mathscr{B}_{-}$, then

$$
\mathbb{P}_{z}(N=\infty)=e^{-\alpha^{\star} z}
$$

where $\alpha^{\star}$ is the unique fixed point of $h(\cdot)$.

Proof: In the setting of Lemma 2 , set $u\left(z_{1}, t, z_{2}\right):=1$ so that $u_{n}(z)=\mathbb{P}_{z}(N>n)$. This entails that $u_{0}(z)=1_{\{z>0\}} \mathbb{P}_{z}(N>0)=1_{\{z>0\}}$. Recall that $\mathbb{P}_{z}(Y(t)=0)=e^{-\lambda_{Y} t}$ if $Y \in \mathscr{C}$, and zero otherwise. Hence we have that for $z>0$ and $Y \in \mathscr{C}$,

$$
\begin{aligned}
u_{1}(z) & =\mathbb{P}\left(\tau_{1}<T_{\lambda, 1}, Y\left(c \tau_{1}\right)>0\right)=\mathbb{E}_{z}\left[e^{-\lambda \tau_{1}} \mathbb{E}_{z}\left[1_{\left\{Y\left(c \tau_{1}\right)>0\right\}} \mid \tau_{1}\right]\right] \\
& =\mathbb{E}_{z}\left[e^{-\lambda \tau_{1}}\left(1-\mathbb{P}_{z}\left(Y\left(c \tau_{1}\right)=0 \mid \tau_{1}\right)\right)\right] \\
& =\mathbb{E}_{z} e^{-\lambda \tau_{1}}-\mathbb{E}_{z} e^{-\left(\lambda+c \lambda_{Y}\right) \tau_{1}}=e^{-h(0) z}-e^{-h(\infty) z} .
\end{aligned}
$$

Likewise, if $Y \notin \mathscr{C}$, then

$$
u_{1}(z)=\mathbb{P}\left(\tau_{1}<T_{\lambda, 1}\right)=e^{-h(0) z} .
$$


For $n=2,3, \ldots$ the stated follows by induction. Concentrate on $Y \notin \mathscr{C} ; Y \in \mathscr{C}$ follows analogously. By Lemma 2,

$$
\begin{aligned}
u_{n}(z) & =\mathbb{E}_{z}\left[e^{-\lambda \tau_{1}} \mathbb{E}_{z}\left[u_{n-1}\left(Y\left(c \tau_{1}\right)\right) \mid \tau_{1}\right]\right] \\
& =\mathbb{E}_{z}\left[e^{-\lambda \tau_{1}} \mathbb{E}_{z}\left[\mathbb{P}_{Y\left(c \tau_{1}\right)}(N>n-1) \mid \tau_{1}\right]\right] \\
& =\mathbb{E}_{z}\left[e^{-\left(\lambda+c \eta\left(h^{n-1}(0)\right)\right) \tau_{1}}\right]=e^{-\psi\left(\lambda+\eta\left(h^{n-1}(0)\right)\right) z}=e^{-h\left(h^{n-1}(0)\right) z}=e^{-h^{n}(0) z} .
\end{aligned}
$$

The claim about $\mathbb{P}_{z}(N=\infty)$ follows from Lemma 1 .

Remark 3 Using the techniques from the proof of Lemma 3, we can also prove

$$
\mathbb{E}_{z} e^{-\alpha Z_{n}} 1_{\{N>n\}}= \begin{cases}e^{-h^{n}(\alpha) z}-e^{-h^{n}(\infty) z} & \text { if } Y \in \mathscr{C} \\ e^{-h^{n}(\alpha) z} & \text { otherwise. }\end{cases}
$$

Conclude from the above lemma that $N$ is not $\mathbb{P}_{z}$-defective if (i) $Y \in \mathscr{C}$, or (ii) $X \in \mathscr{B}_{-} . N$ is $\mathbb{P}_{z}$-defective if (i) $Y \notin \mathscr{C}$, and (ii) $X \notin \mathscr{B}_{-}$. In the following lemma we look at somewhat more refined statements: in specific cases all moments of $N$ exist. We also consider the case in which the initial value is sampled from $B$.

Lemma 4 (i) Suppose that either $Y \in \mathscr{C}$, or $X \in \mathscr{B}_{-}$. Then $\mathbb{E}_{z} e^{t N}<\infty$ for some $t>0$ and thus $\mathbb{E}_{z} N^{p}<\infty$ for all $p>0$.

(ii) Let $\mathbb{E} B$ be finite. Suppose that either (A) $Y \in \mathscr{C}$, or (B) $X \in \mathscr{B}_{-}$, and $\mathbb{P}(B>\epsilon)=1$ for some $\epsilon>0$. Then $\mathbb{E}_{B} e^{t N}<\infty$ for some $t>0$ and thus $\mathbb{E}_{B} N^{p}<\infty$ for all $p>0$.

Proof: When $X \in \mathscr{B}_{-}$, we have that $h^{n}(0) \geq n \psi(\lambda)$ so that the first part of the lemma follows from Lemma 3:

$$
\begin{aligned}
\mathbb{E}_{z} e^{t N} & =1+\left(e^{t}-1\right) \sum_{n=0}^{\infty} e^{t n} \mathbb{P}_{z}(N>n) \\
& \leq 1+\left(e^{t}-1\right) \sum_{n=0}^{\infty} e^{t n} e^{-h^{n}(0) z} \leq 1+\left(e^{t}-1\right) \sum_{n=0}^{\infty} e^{t n} e^{-n \psi(\lambda) z} .
\end{aligned}
$$

When $Y$ is compound Poisson, then we have for each $m \in\{1, \ldots, n\}, n=1,2, \ldots$, that

$$
e^{-h^{n}(0) z}-e^{-h^{n}(\infty) z} \leq\left(h^{n}(\infty)-h^{n}(0)\right) z \leq h^{\prime}\left(h^{m}(0)\right)^{n-m}\left(h^{m}(\infty)-h^{m}(0)\right) z ;
$$

hence if we choose $m$ such that $h^{m}(0)$ is sufficiently close to $\alpha^{\star}$ so that $h^{\prime}\left(h^{m}(0)\right)<1$, the result follows in the same fashion relying on (14).

Now proceed with the second part. When $\mathbb{E} B<\infty$ then for $Y \in \mathscr{C}$ we have that

$$
\mathbb{P}_{B}(N>n) \leq h^{\prime}\left(h^{m}(0)\right)^{n-m}\left(h^{m}(\infty)-h^{m}(0)\right) \mathbb{E} B,
$$

and when $X \in \mathscr{B}_{-}$and $\mathbb{P}(B>\epsilon)=1$, then $\mathbb{P}_{B}(N>n) \leq e^{-h^{n}(0) \epsilon} \leq e^{-n h(0) \epsilon}$ so that for both cases the result follows from the representation (14). 


\section{Existence of stationary distributions}

Applying the results of the previous section, we can present conditions under which it is guaranteed that the embedded process $\left\{Z_{n} \mid n \in \mathbb{N}\right\}$ obeys specific recurrence properties. Later in this section, we also state our result for the (continuous-time) storage process.

Theorem 1 Let $\mathbb{E} B$ be finite. Suppose that either (A) $Y \in \mathscr{C}$, or (B) $X \in \mathscr{B}_{-}$, and $\mathbb{P}(B>\epsilon)=1$ for some $\epsilon>0$. Then $\left\{Z_{n} \mid n \in \mathbb{N}\right\}$ is irreducible, positive Harris recurrent and converges in distribution to its unique stationary distribution.

Proof: Apply Lemma 4. It implies that 0 is a positive recurrent state (regenerative with finite expected regeneration time), and it is accessible from any state. For each $z \geq 0$,

$$
\mathbb{P}_{z}\left(Z_{1}=0\right) \geq \mathbb{P}_{z}\left(\tau_{1}<T_{\lambda, 1}\right)=e^{-h(0) z}>0,
$$

entailing that the regeneration epochs are aperiodic. Thus, a limiting distribution exists and it is the stationary distribution.

Despite the fact that $N$ can be $\mathbb{P}_{z}$-defective, $\sum_{n=1}^{N} \tau_{n}$ has always a bounded mean (for a given initial storage level $z$ ). This is shown in the next lemma, which also considers the case of a random initial storage level.

Lemma 5 (i) $\mathbb{E}_{z} \sum_{n=1}^{N} \tau_{n}<\infty$ for all $z>0$.

(ii) Let $B$ be a positive random variable with $\mathbb{E} B<\infty$. If either $X \in \mathscr{U}$, or $X \in \mathscr{B}_{+}$, then $\mathbb{E}_{B} \sum_{n=1}^{N} \tau_{n}<\infty$.

(iii) Let $B$ be a positive random variable with $\mathbb{E} B<\infty$ and $\mathbb{P}(B>\epsilon)=1$ for some $\epsilon>0$. If $X \in \mathscr{B}_{-}$, then $\mathbb{E}_{B} \sum_{n=1}^{N} \tau_{n}<\infty$.

Proof: With $u\left(z_{1}, t, z_{2}\right)=t$ in Lemma 2, it is seen that $u_{0}(z)=E_{z} \tau_{1}=z \psi^{\prime}(0)=z / \varphi^{\prime}(0)$, which also holds for $z=0$. Trivially,

$$
\mathbb{E}_{z} \sum_{n=1}^{N} \tau_{n}=\mathbb{E}_{z} \sum_{n=0}^{N-1} \tau_{n+1}=\mathbb{E}_{z} \sum_{n=0}^{\infty} \tau_{n+1} 1_{\{N>n\}}=\sum_{n=0}^{\infty} u_{n}(z) .
$$

With $a_{0}=0$ and $b_{0}=1 / \varphi^{\prime}(0)$, let us assume by induction that $u_{n-1}(z)=b_{n-1} z e^{-a_{n-1} z}$ for $n \geq 1 ; a_{n}$ and $b_{n}$ will be specified later. Then, due to Lemma 2 and the induction hypothesis,

$$
\begin{aligned}
u_{n}(z) & =\mathbb{E}_{z}\left[e^{-\lambda \tau_{1}} \mathbb{E}_{z}\left[u_{n-1}\left(Y\left(c \tau_{1}\right)\right) \mid \tau_{1}\right]\right] \\
& =\mathbb{E}_{z}\left[e^{-\lambda \tau_{1}} \mathbb{E}_{z}\left[b_{n-1} Y\left(c \tau_{1}\right) e^{-a_{n-1} Y\left(c \tau_{1}\right)} \mid \tau_{1}\right]\right] .
\end{aligned}
$$

It is elementary that

$$
\mathbb{E}_{z}\left[Y\left(c \tau_{1}\right) e^{-a_{n-1} Y\left(c \tau_{1}\right)} \mid \tau_{1}\right]=-\frac{\mathrm{d}}{\mathrm{d} a_{n-1}} E_{z} e^{-\left(\lambda+c \eta\left(a_{n-1}\right)\right) \tau_{1}}=-\frac{\mathrm{d}}{\mathrm{d} a_{n-1}} e^{-c \eta\left(a_{n-1}\right) \tau_{1}} .
$$


Hence,

$$
\begin{aligned}
u_{n}(z) & =b_{n-1}\left(-\frac{\mathrm{d}}{\mathrm{d} a_{n-1}} \mathbb{E}_{z} e^{-\left(\lambda+c \eta\left(a_{n-1}\right)\right) \tau_{1}}\right)=b_{n-1}\left(-\frac{\mathrm{d}}{\mathrm{d} a_{n-1}} e^{-\psi\left(\lambda+c \eta\left(a_{n-1}\right)\right) z}\right) \\
& =b_{n-1}\left(-\frac{\mathrm{d}}{\mathrm{d} a_{n-1}} e^{-h\left(a_{n-1}\right) z}\right)=b_{n-1} h^{\prime}\left(a_{n-1}\right) z e^{-h\left(a_{n-1}\right) z} .
\end{aligned}
$$

This implies that we should choose $a_{n}:=h\left(a_{n-1}\right)=h^{n}(0)$ for $n=0,1, \ldots$, and

$$
b_{n}:=b_{n-1} h^{\prime}\left(h^{n-1}(0)\right)=\frac{\prod_{i=0}^{n-1} h^{\prime}\left(h^{i}(0)\right)}{\varphi^{\prime}(0)},
$$

for $n=1,2, \ldots$. It now follows that

$$
u_{n}(z)=\frac{\prod_{i=0}^{n-1} h^{\prime}\left(h^{i}(0)\right)}{\varphi^{\prime}(0)} z e^{-h^{n}(0) z} .
$$

Now let us verify whether the $u_{n}(z)$ are indeed summable for all $z>0$; we will use the sufficient condition that $\lim \sup _{n \rightarrow \infty} u_{n+1}(z) / u_{n}(z)$ is strictly smaller than 1 . Distinguish, as in Lemma 1, between two cases. First suppose that $X \in \mathscr{U}$, or $X \in \mathscr{B}_{+}$. Then Lemma 1 entails the existence of a fixed point $\alpha^{\star}$, and hence the stated follows: as $n \rightarrow \infty$,

$$
\frac{u_{n+1}(z)}{u_{n}(z)}=h^{\prime}\left(h^{n}(0)\right) e^{-\left(h^{n+1}(0)-h^{n}(0)\right) z} \rightarrow h^{\prime}\left(\alpha^{\star}\right) e^{-\left(\alpha^{\star}-\alpha^{\star}\right) z}=h^{\prime}\left(\alpha^{\star}\right)<1 .
$$

Now consider the other case: $X \in \mathscr{B}_{-}$. Then $h^{n-1}(0) \rightarrow \infty$ as $n \rightarrow \infty$ and we recall that $\psi^{\prime}(\alpha)=1 / \varphi^{\prime}(\psi(\alpha)) \downarrow 1 / b$ as $\alpha \rightarrow \infty$. Therefore,

$$
\begin{aligned}
h(\alpha)-h(0) & =\psi(\lambda+c \eta(\alpha))-\psi(\lambda) \\
& \geq \psi^{\prime}(\lambda+c \eta(\alpha)) c \eta(\alpha) \geq \frac{c}{b}(\eta(\alpha)-a \alpha)+\frac{c a}{b} \alpha,
\end{aligned}
$$

so that

$$
h(\alpha)-\alpha \geq h(0)+\frac{c}{b}(\eta(\alpha)-a \alpha)+\left(\frac{c a}{b}-1\right) \alpha,
$$

where we recall that $\eta(\alpha)-a \alpha \rightarrow \nu_{Y}(0, \infty) \equiv \lambda_{Y}$ as $\alpha \rightarrow \infty$. Now,

$$
\begin{aligned}
\frac{u_{n+1}(z)}{u_{n}(z)} & =h^{\prime}\left(h^{n}(0)\right) e^{-\left(h\left(h^{n}(0)\right)-h^{n}(0)\right) z} \\
& \leq h^{\prime}\left(h^{n}(0) \exp \left(-\left(h(0)+\frac{c}{b}\left(\eta\left(h^{n}(0)\right)-a h^{n}(0)\right)+\left(\frac{c a}{b}-1\right) h^{n}(0)\right) z\right)\right. \\
& \rightarrow \begin{cases}0 & \text { if either } b<c a \text { or } \lambda_{Y}=\infty \\
\exp \left(-\left(h(0)+\frac{c}{b} \lambda_{Y}\right) z\right)<1 & \text { if both } b=c a \text { and } \lambda_{Y}<\infty\end{cases}
\end{aligned}
$$

as $n \rightarrow \infty$; here we recall that when $X \in \mathscr{B}_{-}$, then $h^{n}(0) \rightarrow \infty$, and thus $h^{\prime}\left(h^{n}(0)\right) \rightarrow c a / b$ as $n \rightarrow \infty$. Conclude that also in this case $\sum_{n=0}^{\infty} u_{n}(z)<\infty$; this ends the proof of part (i).

Now consider part (ii). When either $X \in \mathscr{U}$ or $X \in \mathscr{B}_{+}$, we have that $u_{n}(z) \leq b_{n} z$ and thus $\mathbb{E} u_{n}(B) \leq b_{n} \mathbb{E} B$ and since $\sum_{n=1}^{\infty} b_{n}<\infty$ as $h^{\prime}\left(\alpha^{\star}\right)<1$, then $\sum_{n=0}^{\infty} \mathbb{E} u_{n}(B)<\infty$.

Part (iii) is proven as follows. When $X \in \mathscr{B}_{-}$and $\mathbb{P}(B>\epsilon)=1$ we have from (16) that $u_{n}(B) \leq(B / \epsilon) u_{n}(\epsilon)$, so that the result follows from the fact that $\mathbb{E} B<\infty$ and that $\sum_{n=0}^{\infty} u_{n}(\epsilon)<\infty$. 
Theorem 2 Assume $\mathbb{E} B<\infty$. Assume that if $X \in \mathscr{B}_{-}$, then $\mathbb{P}(B>\epsilon)=1$ for some $\epsilon>0$. Then the (continuous-time) storage process described is regenerative where the regeneration epoch has a density and a finite mean. Thus the process is stable and has a unique stationary distribution which is also its limiting distribution.

Proof: Consider the epochs that the process begins with a jump (the size of which being distributed like $B$ ) after a previous cycle. It is clear that these times are regeneration epochs for the continuous-time process. The expected regeneration time starting from level $B$ is

$$
\mathbb{E}_{B} \sum_{n=1}^{N}\left(\tau_{n}\left(1+c 1_{\left\{\tau_{n}<T_{\lambda, n}\right\}}\right)\right) \leq(1+c) \mathbb{E}_{B}\left(\sum_{n=1}^{N} \tau_{n}\right)=(1+c) \sum_{n=1}^{\infty} \mathbb{E} u_{n}(B) .
$$

As shown in Lemma 5, the right-hand side is finite. The regeneration interval clearly has a density. One can see this, e.g., by observing that it is a sum of two independent random variables, one of which is exponential (the time until the jump initiating the next cycle).

Example 1 In Lemma 3 we observed the perhaps somewhat unexpected phenomenon that the random variable $N$ (i.e., the number of cycles per regeneration period) can be defective. On the other hand, the duration of the regeneration period still has finite mean (Thm. 2). To demonstrate this in a simple case, assume that $X(t)=-t$ and $Y(t)=t$. We start at an arbitrary storage level $z$ at time 0 . Then $a=b=1$, and also $\varphi(\alpha)=\alpha, \psi(\alpha)=\alpha, \eta(\alpha)=\alpha$, and hence

$$
h(\alpha)=\psi(\lambda+c \eta(\alpha))=\lambda+c \alpha .
$$

Thus, if $c<1$, then we are in the situation that $X \in \mathscr{B}_{+}$, and hence, according to Lemma 3, the number of cycles $N$ should be $\mathbb{P}_{z}$-defective; if $c \geq 1$, we have that $X \in \mathscr{B}_{-}$and hence $N$ is finite $\mathbb{P}_{z}$-almost-surely. Let us verify these facts.

If $c<1$, then $\alpha^{\star}=\lambda /(1-c)$, and $h^{\prime}\left(\alpha^{\star}\right)=c<1$. We see from Lemma 3 that

$$
\mathbb{P}_{z}(N>n)=e^{-h^{n}(0) z}=\exp \left(-\lambda \frac{1-c^{n}}{1-c}\right),
$$

so that indeed $\mathbb{P}_{z}(N=\infty)=e^{-\alpha^{\star} z}$, as expected. This could also be found directly in this case. Note that, on $\{N>n\}$, it holds that $\tau_{n+1}=z c^{n}$. Therefore,

$$
\mathbb{P}(N=\infty)=\mathbb{P}\left(\forall n \in \mathbb{N}: T_{\lambda, n}>\tau_{n}\right)=\prod_{n=1}^{\infty} \mathbb{P}\left(T_{\lambda}>z c^{n-1}\right),
$$

which also gives $e^{-\alpha^{\star} z}$.

On $\{N>n\}$, we clearly have that $Z_{n}=c^{n} z$. It is also easy to check that in this case

$$
b_{n}=b_{n-1} h^{\prime}\left(h^{n}(0)\right)=c b_{n-1}=\frac{c^{n}}{\varphi^{\prime}(0)}=c^{n},
$$

using the notation of the proof of Lemma 5. It implies that, cf. (16),

$$
u_{n}(z)=c^{n} z \exp \left(-\lambda \frac{1-c^{n}}{1-c} z\right),
$$


which immediately leads to $\sum_{n=0}^{\infty} u_{n}(z) \leq z /(1-c)$. This means that the mean length of a regeneration period is bounded above by $z(1+c) /(1-c)$, cf. the proof of Thm. 2 .

For $c>1$ (the case $c=1$ can be dealt with similarly) it is readily verified that (17) still applies, and hence $\mathbb{P}_{z}(N>n) \rightarrow 0$ as $n \rightarrow \infty$. In fact, $N$ has an exponentially bounded tail, due to Lemma 4 . Let us consider the case that $B$ is deterministically equal to 1 . Due to Thm. 1 we know that the $Z_{n}$ have a proper stationary distribution. Let us try to determine this. With $Z$ being the stationary storage level, from (7) it is seen that $F(\alpha):=\mathbb{E} e^{-\alpha Z}$ satisfies

$$
F(\alpha)=1+F(\lambda+c \alpha)-F(\lambda)+e^{-\lambda}\left(e^{-c \alpha}-1\right) \pi_{0},
$$

with $\pi_{0}:=\mathbb{P}(Z=0)$. Letting $\alpha \rightarrow \infty$ yields $\pi_{0}=(1-F(\lambda)) e^{\lambda}$ (use that $F(\infty)=\pi_{0}$ ). We obtain the equation

$$
F(\alpha)=F(\lambda+c \alpha)+g(\alpha),
$$

with $g(\alpha):=(1-F(\lambda)) e^{-c \alpha}$. Successive iteration yields after $n$ steps (where empty sums are interpreted as 0 )

$$
F(\alpha)=F\left(\lambda \sum_{i=0}^{n} c^{i}+c^{n+1} \alpha\right)+\sum_{j=0}^{n} g\left(\lambda \sum_{i=0}^{j-1} c^{i}+c^{j} \alpha\right) .
$$

Now let $n \rightarrow \infty$ to obtain (use that $c>1$ )

$$
F(\alpha)=(1-F(\lambda)) e^{\lambda}+(1-F(\lambda)) \sum_{j=0}^{\infty} \exp \left(-c\left(\lambda \sum_{i=0}^{j-1} c^{i}+c^{j} \alpha\right)\right) .
$$

Finally, $F(\lambda)$ can be determined by inserting $\alpha=0$. It yields, with $s_{k}:=\exp \left(-\lambda \sum_{i=1}^{k-1} c^{i}\right)$,

$$
F(\alpha)=\sum_{j=0}^{\infty} p_{j} e^{-\alpha q_{j}}, \text { where } p_{0}:=\frac{e^{\lambda}}{e^{\lambda}+\sum_{k=1}^{\infty} s_{k}}, p_{j}:=\frac{s_{j}}{e^{\lambda}+\sum_{k=1}^{\infty} s_{k}} \quad(j=1,2 \ldots)
$$

and $q_{0}:=0, q_{j}:=c^{j}$ for $j=1,2 \ldots$ This result can be interpreted as: $Z$ is zero with probability $\pi_{0}=p_{0}$, and has value $c^{j}$ with probability $p_{j}$ for $j=1,2 \ldots$

\section{Computation of stationary LST s}

In this section we compute the LST of the stationary distribution of $\left\{Z_{n} \mid n \in \mathbb{N}\right\}$ for the sitatutions that either $X \in \mathscr{B}_{-}$or $Y \in \mathscr{C}$. Then we focus on the stationary distribution of the contiuous-time storage process.

Let us start by considering the stationary distribution of $\left\{Z_{n} \mid n \in \mathbb{N}\right\}$ for $X \in \mathscr{B}_{-}$. We assume that there is an $\epsilon>0$ such that $\mathbb{P}(B>\epsilon)=1$, so that, by virtue of Thm. 1 we have a proper stationary distribution (equalling the limiting distribution). It is noted that Example 1 (for $c>1$ ) deals with such a case, and in fact the procedure followed there can 
be applied here as well. Recall (7), with $F(\alpha):=\mathbb{E} e^{-\alpha Z}$ and $\pi_{0}:=\mathbb{P}(Z=0)$. Letting $\alpha \rightarrow \infty$ in (7), we find

$$
\pi_{0}=\frac{1-F(\psi(\lambda))}{\beta(\psi(\lambda))}
$$

where it is used that $h(\infty)=\infty$ and $\beta(h(\infty))=0$ (use $\mathbb{P}(B=0)=0$ ), and hence (7) becomes $F(\alpha)=F(h(\alpha))+\pi_{0} \beta(h(\alpha))$. Straightforward iteration of this relation gives, after $n-1$ steps,

$$
F(\alpha)=F\left(h^{n}(\alpha)\right)+\pi_{0} \sum_{i=1}^{n} \beta\left(h^{i}(\alpha)\right) .
$$

Now let $n \rightarrow \infty$. Realizing that $h^{n}(\alpha) \rightarrow \infty$ as $n \rightarrow \infty$ (see Lemma 1), we obtain

$$
F(\alpha)=\pi_{0}\left(1+\sum_{i=1}^{\infty} \beta\left(h^{i}(\alpha)\right)\right) .
$$

The requirement $F(0)=1$ yields the following result.

Theorem 3 Let $\mathbb{E} B<\infty$ and $\mathbb{P}(B>\epsilon)=1$ for some $\epsilon>0$. Then for $X \in \mathscr{B}_{-}$,

$$
\mathbb{E} e^{-\alpha Z}=\frac{1+\sum_{i=1}^{\infty} \beta\left(h^{i}(\alpha)\right)}{1+\sum_{i=1}^{\infty} \beta\left(h^{i}(0)\right)},
$$

where all the sums involved are finite.

We note that the finiteness of the sums involved in (18) follows from Lemmas 3 and 4, as

$$
\mathbb{E}_{B} N=1+\sum_{i=1}^{\infty} \beta\left(h^{i}(0)\right)
$$

is finite when $\mathbb{E} B<\infty$ and $\mathbb{P}(B>\epsilon)=1$ for some $\epsilon>0$.

We now consider the other situation in which $N$ is not defective, viz. $Y \in \mathscr{C}$. Again, due to Thm. 1 we have a proper stationary distribution which equals the limiting distribution. It is important to note that now $h(\infty)=\psi\left(\lambda+c \lambda_{Y}\right)<\infty$. Relying on Lemma 1, there is an $\alpha^{\star}$ such that $h\left(\alpha^{\star}\right)=\alpha^{\star}$. Plugging this $\alpha^{\star}$ in (7), and substracting the resulting relation from (7), we obtain

$$
F(\alpha)=F(h(\alpha))+\left(\beta(h(\alpha))-\beta\left(h\left(\alpha^{\star}\right)\right)\right) \pi_{0} .
$$

Iteration leads, after $n-1$ steps, to

$$
F(\alpha)=F\left(h^{n}(\alpha)\right)+\pi_{0} \sum_{i=1}^{n}\left(\beta\left(h^{i}(\alpha)\right)-\beta\left(\alpha^{\star}\right)\right) .
$$

Now letting $\alpha \rightarrow \infty$, and subtracting the resulting relation from (19), we obtain

$$
F(\alpha)=\pi_{0}+F\left(h^{n}(\alpha)\right)-F\left(h^{n}(\infty)\right)+\pi_{0} \sum_{i=1}^{n}\left(\beta\left(h^{i}(\infty)\right)-\beta\left(\alpha^{\star}\right)\right) .
$$

Now send $n \rightarrow \infty$, and solve $\pi_{0}$ by imposing $F(0)=1$, and we obtain the following result. 
Theorem 4 Let $\mathbb{E} B<\infty$. Then for $Y \in \mathscr{C}$,

$$
\mathbb{E} e^{-\alpha Z}=\frac{1+\sum_{i=1}^{\infty}\left(\beta\left(h^{i}(\alpha)\right)-\beta\left(h^{i}(\infty)\right)\right)}{1+\sum_{i=1}^{\infty}\left(\beta\left(h^{i}(0)\right)-\beta\left(h^{i}(\infty)\right)\right)}
$$

where all the sums involved are finite.

The remainder of this section is devoted to determining the LST of the stationary distribution of the continuous-time storage process, which exists, under mild conditions on $B$, due to Thm. 2.

Introduce the exponential of the storage process, taken over the $(n+1)$ th cycle and with $n<N$ : with $T_{0} \equiv 0$ and $T_{n}:=(1+c) \sum_{i=1}^{n} \tau_{i}$ for $n=1,2, \ldots$,

$$
\begin{aligned}
g_{n}(z, \alpha) & =\mathbb{E}_{z}\left[\int_{T_{n}}^{T_{n}+\tau_{n+1}} e^{-\alpha\left(Z_{n}+X(t)-X\left(T_{n}\right)\right)} \mathrm{d} t\right. \\
& \left.+\int_{T_{n}+\tau_{n+1}}^{T_{n}+(1+c) \tau_{n+1}} e^{-\alpha\left(Y(t)-Y\left(T_{n}+\tau_{n+1}\right)\right.} \mathrm{d} t 1_{\left\{\tau_{n+1}<T_{\lambda, n+1}\right\}}\right] 1_{\{N>n\}} .
\end{aligned}
$$

Lemma 6 For $n=1,2, \ldots$,

$$
g_{n}(z, \alpha)=\frac{e^{-h^{n}(0) z}-e^{-h^{n}(\alpha) z}}{\varphi(\alpha)}+\frac{e^{-h^{n+1}(0) z}-e^{-h^{n+1}(\alpha) z}}{\eta(\alpha)},
$$

and consequently

$$
g_{n}(z, 0)=\frac{z e^{-h^{n}(0) z} \prod_{i=0}^{n-1} h^{\prime}\left(h^{i}(0)\right)}{\varphi^{\prime}(0)}+\frac{z e^{-h^{n+1}(0) z} \prod_{i=0}^{n} h^{\prime}\left(h^{i}(0)\right)}{\eta^{\prime}(0)} .
$$

Proof: We first observe that

$$
\mathbb{E}_{z} e^{-\alpha X\left(\tau_{1} \wedge t\right)}-e^{-\alpha z}=\varphi(\alpha) \mathbb{E}_{z} \int_{0}^{\tau_{1} \wedge t} e^{-\alpha X(s)} \mathrm{d} s \rightarrow \varphi(\alpha) \mathbb{E}_{z} \int_{0}^{\tau_{1}} e^{-\alpha X(s)} \mathrm{d} s,
$$

as $t \rightarrow \infty$; here the equality is due to [6], and the convergence due to the monotone convergence theorem. Realizing that $X\left(\tau_{1} \wedge t\right) \geq 0 \mathbb{P}_{z}$-almost-surely for all $t$, we have by bounded convergence that the left-hand side of (20) converges to $1-e^{-\alpha z}$, and hence

$$
\mathbb{E}_{z} \int_{0}^{\tau_{1}} e^{-\alpha X(s)} \mathrm{d} s=\frac{1-e^{-\alpha z}}{\varphi(\alpha)} .
$$

Also, for each fixed $t$ we have that

$$
\mathbb{E}_{z} \int_{0}^{c t} e^{-\alpha Y(s)} \mathrm{d} s=\int_{0}^{c t} e^{-\eta(\alpha) s} \mathrm{~d} s=\frac{1-e^{-c \eta(\alpha) t}}{\eta(\alpha)},
$$

and, since $Y$ and $T_{\lambda, 1}$ are independent,

$$
\mathbb{E}_{z} \int_{0}^{c t} e^{-\alpha Y(s)} \mathrm{d} s 1_{\left\{t<T_{\lambda, 1}\right\}}=\frac{e^{-\lambda t}-e^{-(\lambda+c \eta(\alpha)) t}}{\eta(\alpha)} .
$$


Therefore upon conditioning on $\tau_{1}$ and then unconditioning,

$$
\mathbb{E}_{z} \int_{0}^{c \tau_{1}} e^{-\alpha Y(s)} \mathrm{d} s 1_{\left\{\tau_{1}<T_{\lambda, 1}\right\}}=\frac{\mathbb{E}_{z} e^{-\lambda \tau_{1}}-\mathbb{E}_{z} e^{-(\lambda+c \eta(\alpha)) \tau_{1}}}{\eta(\alpha)}=\frac{e^{-h(0) z}-e^{-h(\alpha) z}}{\eta(\alpha)} .
$$

By conditioning on $\tau_{1}, \ldots, \tau_{n}, Z_{1}, \ldots, Z_{n}$ and applying the strong Markov property for Lévy processes it readily follows that

$$
g_{n}(z, \alpha)=\mathbb{E}_{z}\left[\frac{1-e^{-\alpha Z_{n}}}{\varphi(\alpha)}+\frac{e^{-h(0) Z_{n}}-e^{-h(\alpha) Z_{n}}}{\eta(\alpha)}\right] 1_{\{N>n\}}
$$

where

$$
g_{0}(z, \alpha)=\frac{1-e^{-\alpha z}}{\varphi(\alpha)}+\frac{e^{-h(0) z}-e^{-h(\alpha) z}}{\eta(\alpha)} .
$$

From (13) the result now follows.

We now obtain the following result.

Theorem 5 The LST of the stationary and limiting distribution of the described storage process is given by

$$
\frac{\delta^{-1}+\sum_{n=0}^{\infty} \mathbb{E} g_{n}(B, \alpha)}{\delta^{-1}+\sum_{n=0}^{\infty} \mathbb{E} g_{n}(B, 0)}
$$

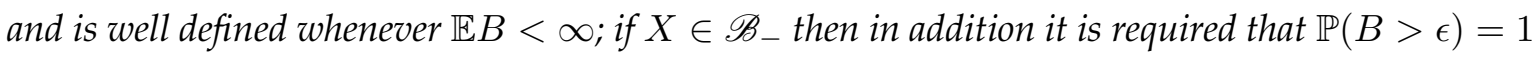
for some $\epsilon>0$.

Example 2 Let us return to the model of Example 1, with $B$ being deterministically equal to 1 . It can be verified that $h^{n}(\alpha)=\lambda \sum_{i=0}^{n-1} c^{i}+c^{n} \alpha$, which leads to

$$
\mathbb{E} g_{n}(B, \alpha) \equiv \mathbb{E} g_{n}(1, \alpha)=\alpha^{-1} \exp \left(-\sum_{i=0}^{n-1} c^{i}\right) \cdot\left(1-e^{-c^{n} \alpha}+e^{-\lambda c^{n}}\left(1-e^{-c^{n+1} \alpha}\right)\right),
$$

and hence

$$
\mathbb{E} g_{n}(B, 0) \equiv \mathbb{E} g_{n}(1,0)=c^{n} \exp \left(-\sum_{i=0}^{n-1} c^{i}\right) \cdot\left(1+c e^{-\lambda c^{n}}\right) .
$$

Then the stationary distribution follows from (21). This holds irrespective of the value of $c>0$ (that is, it does not matter whether $c<1$ or $c \geq 1$ ).

Remark 4 Let us conclude this section with some words on the case where $Y \in \mathscr{C}$, and consider a few special cases. Suppose that $Y \in \mathscr{C}$ with rate $\lambda_{Y}$ and jump distribution $F_{Y}(t)=\nu_{Y}((0, t])$. A special case of this model is the sitation where $\lambda_{Y} \equiv \delta$ and the distribution corresponding to $F_{Y}(\cdot)$ equalling the distribution of $B$. The latter model is equivalent to the model where once $X$ hits the level zero, then either (i) $\tau_{1} \geq T_{\lambda, 1}$, and we wait for the first 
jump epoch of $Y$ before switching back to $X$, or (ii) $\tau_{1}<T_{\lambda, 1}$, and we wait for the maximum between $(1+c) \tau_{1}$ and the first jump epoch of $Y$ before switching back to $X$.

Yet another special case is where $X$ behaves like $Y(t)-b t$, which means that the model becomes equivalent to one with compound Poisson input, and a service rate alternating between $b$ and 0 . In this particular case, to be interpreted as an M/G/1 queue with dependence between busy and idle periods, it does not matter whether we revert to $X$ at the beginning of the exponential time that leads to the first arrival in a regeneration epoch, or at the arrival epoch itself (as was assumed throughout). Indeed, for this latter case we have that $\eta(\alpha)=\delta(1-\beta(\alpha))$ and $\varphi(\alpha)=b \alpha-\eta(\alpha)$. Therefore, for this case $h\left(\alpha^{\star}\right)=\psi\left(\lambda+c \eta\left(\alpha^{\star}\right)\right)=\alpha^{\star}$ is equivalent to

$$
b \alpha^{\star}-(1+c) \delta\left(1-\beta\left(\alpha^{\star}\right)\right)=\lambda .
$$

That is, if we consider the process $z+(1+c) Y(t)-b t$ and let $\tau^{\star}$ be the first instance it hits zero (which is infinitely large if it never happens), then $\mathbb{P}_{z}\left(\tau^{\star}<T_{\lambda}\right)=\mathbb{E}_{z} e^{-\lambda \tau^{\star}}=e^{-\alpha^{\star} z}$. Note that this holds even when $b<(1+c) \delta \cdot \mathbb{E} B$, in which case there is a positive probability that zero is never hit. We note that the process $(z+(1+c) Y(t)-b t) / b$ is the net input process in an $\mathrm{M} / \mathrm{G} / 1$ queue with arrival rate $(1+c) \delta / b$ and service times distributed like $B$.

\section{The distribution of the regeneration period}

In this section we discuss the steady-state distribution of the regeneration time, viz., the time between two successive time epochs in which the process starts from an independent initial position distributed like $B$, cf. Remark 1 . In this section we take for simplicity $\delta=\infty$ (but it is noted that the results can be adapted in a straightforward way to make them applicable for $\delta \in(0, \infty)$, too).

Let us first assume that we start off at a given initial storage level $z$, and consider the LST $f(z):=\mathbb{E}_{z} e^{-\alpha R}$ of the regeneration period $R$. As before, we can decompose this by conditioning:

$$
\begin{aligned}
& f(z)=\mathbb{E}_{z}\left[e^{-\alpha \tau_{1}} 1_{\left\{\tau_{1} \geq T_{\lambda, 1}\right\}}\right]+\mathbb{E}_{z}\left[e^{-\alpha(1+c) \tau_{1}} \mathbb{E}_{Y\left(c \tau_{1}\right)}\left[e^{-\alpha R}\right] 1_{\left\{\tau_{1}<T_{\lambda, 1}, Y\left(c \tau_{1}\right)>0\right\}}\right] \\
&+ \mathbb{E}_{z}\left[e^{-\alpha(1+c) \tau_{1}} 1_{\left\{\tau_{1}<T_{\lambda, 1}, Y\left(c \tau_{1}\right)=0\right\}}\right] \\
&=\mathbb{E}_{z}\left[e^{-\alpha \tau_{1}}\right]-\mathbb{E}_{z}\left[e^{-(\alpha+\lambda) \tau_{1}}\right]+\mathbb{E}_{z}\left[e^{-\alpha(1+c) \tau_{1}} f\left(Y\left(c \tau_{1}\right)\right) 1_{\left\{\tau_{1}<T_{\lambda, 1}, Y\left(c \tau_{1}\right)>0\right\}}\right] \\
&+\mathbb{E}_{z}\left[e^{-\alpha(1+c) \tau_{1}} 1_{\left\{\tau_{1}<T_{\lambda, 1}, Y\left(c \tau_{1}\right)=0\right\}}\right] \\
&=e^{-\psi(\alpha) z}-e^{-\psi(\alpha+\lambda) z}+\mathbb{E}_{z}\left[e^{-(\alpha(1+c)+\lambda) \tau_{1}}\left(1-e^{-c \lambda_{Y} \tau_{1}}\right) f\left(Y\left(c \tau_{1}\right)\right) \mid \tau_{1}\right] \\
&+e^{-\psi\left(\alpha(1+c)+\lambda+c \lambda_{Y}\right) z}
\end{aligned}
$$

where the last term in (22) can be ignored if $Y \notin \mathscr{C}$, and then also the term $e^{-c \lambda_{Y} \tau_{1}}$ disappears. In the sequel we assume for ease that $Y \notin \mathscr{C}$, so that $\{N>n\}$ coincides with 
$\left\{T_{\lambda, i} \geq \tau_{i}\right\}$ for all $i=1, \ldots, n$. Let us iterate formula (22). Realizing that

$$
\begin{aligned}
\mathbb{E}_{z} & {\left[e^{-(\alpha(1+c)+\lambda) \tau} e^{-\psi(\alpha) Y(c \tau)} \mid \tau\right]=\int_{0}^{\infty} e^{-(\alpha(1+c)+\lambda) t} \mathbb{E}\left[e^{-\psi(\alpha) Y(c t)}\right] \mathrm{d} \mathbb{P}\left(\tau_{z}<t\right) } \\
& =\int_{t=0}^{\infty} e^{-(\alpha(1+c)+\lambda) t} e^{-\eta(\psi(\alpha)) c t} \mathrm{~d} \mathbb{P}\left(\tau_{z}<t\right)=\exp (-\psi[\alpha(1+c)+\lambda+c \eta(\psi(\alpha))] z),
\end{aligned}
$$

and defining $k^{0}(\alpha):=\psi(\alpha), m^{0}(\alpha):=\psi(\alpha+\lambda)$, and

$$
\begin{aligned}
k(x) & :=\psi(\alpha(1+c)+\lambda+c \eta(x)), \\
k^{n+1}(\alpha) & :=k\left(k^{n}(\alpha)\right), \quad n=0,1, \ldots, \\
m^{n+1}(\alpha) & :=k\left(m^{n}(\alpha)\right), \quad n=0,1, \ldots,
\end{aligned}
$$

we thus obtain that

$$
\mathbb{E}_{z} e^{-\alpha R}=f(z)=\sum_{n=0}^{\infty}\left(e^{-k^{n}(\alpha) z}-e^{-m^{n}(\alpha) z}\right) .
$$

We notice that the $n$th term in (23) can be interpreted as

$$
\mathbb{E} \exp \left(-\alpha\left((1+c) \sum_{i=1}^{n} \tau_{i}+\tau_{n+1}\right)\right) 1_{\{N=n+1\}},
$$

that is, the contribution to $R$ due to regeneration periods consisting of $n+1$ cycles ( $n=$ $0,1, \ldots)$. Now starting according to a random variable $B$, Eq. (23) leads to the following result.

Theorem 6 If $Y \notin \mathscr{C}$, then the LST of the duration of the regeneration period is given by

$$
\mathbb{E} e^{-\alpha R}=\sum_{n=0}^{\infty}\left(\beta\left(k^{(n)}(\alpha)\right)-\beta\left(m^{(n)}(\alpha)\right)\right) .
$$

Example 3 We return to the setting of Example 1, where $X(t)=-t, Y(t)=t$, and a generally distributed $B$. We first derive the LST of the regeneration period directly, and then we verify that the method outlined in this section yields the same result.

With $R_{z}$ the length of a cycle starting at level $z$ at the beginning of a busy period,

$$
R_{z}=(1+c) \sum_{i=1}^{N-1} \tau_{i}+\tau_{N}=(1+c) \sum_{i=1}^{N-1} c^{i-1} z+c^{N-1} z=\left((1+c) \frac{1-c^{N-1}}{1-c}+c^{N-1}\right) z
$$

We found earlier that

$$
\mathbb{P}_{z}(N=n)=\exp \left(-\lambda \frac{1-c^{n-1}}{1-c} z\right)-\exp \left(-\lambda \frac{1-c^{n}}{1-c} z\right), \quad n=1,2, \ldots .
$$

Hence, from the preceding two equations,

$$
\mathbb{E}_{z} e^{-\alpha R}=\sum_{n=1}^{\infty} \mathbb{P}_{z}(N=n) \exp \left(-\alpha\left[(1+c) \frac{1-c^{n-1}}{1-c}+c^{n-1}\right] z\right) .
$$


We find, for $z$ distributed like $B$,

$$
\begin{aligned}
\mathbb{E} e^{-\alpha R}=\sum_{n=0}^{\infty} \beta\left(\lambda \frac{1-c^{n}}{1-c}+\alpha\left[(1+c) \frac{1-c^{n}}{1-c}+c^{n}\right]\right) & \\
- & \sum_{n=0}^{\infty} \beta\left(\lambda \frac{1-c^{n}}{1-c}+\lambda c^{n}+\alpha\left[(1+c) \frac{1-c^{n}}{1-c}+c^{n}\right]\right) .
\end{aligned}
$$

It is readily checked that this result matches with what would be obtained by directly applying Thm. 6: Eq. (25) agrees with Eq. (26), with

$$
k^{n}(\alpha)=(\alpha(1+c)+\lambda) \frac{1-c^{n}}{1-c}+\alpha c^{n}, \quad m^{n}(\alpha)=k^{n}(\alpha)+\lambda c^{n} .
$$

\section{Acknowledgment}

The research of O. Boxma and M. Mandjes has been partly funded by the Dutch BSIK/BRICKS (Basic Research in Informatics for Creating the Knowledge Society) project. O. Kella's research is partially supported by grant No. 964/06 from the Israel Science Foundation and the Vigevani Chair in Statistics.

\section{References}

[1] Bertoin, J. (1996). Léoy Processes, Cambridge University Press, Cambridge.

[2] Boxma, O.J., O. Kella and M. Mandjes (2007). On a generic class of Lévy-driven vacation models. EURANDOM Report 2007-057.

[3] Boxma, O.J., M. Mandjes and O. Kella (2007). On a queueing model with interruptions. EURANDOM Report 2007-024. To appear in Probability in the Engineering and Informational Sciences.

[4] Doshi, B.T. (1986). Queueing systems with vacations - a survey. Queueing Systems 1, 29-66.

[5] Doshi, B.T. (1990). Single server queues with vacations. In: Stochastic Analysis of Computer and Communication Systems. H. Takagi (ed.). North-Holland Publ. Co., Amsterdam, pp. 217-265.

[6] Kella, O. and W. Whitt (1992). Useful martingales for stochastic storage processes with Levy input. Journal of Applied Probability 29, 396-403.

[7] Kyprianou, A.E. (2006). Introductory Lectures on Fluctuations of Lévy Processes with Applications, Springer, Berlin.

[8] Prabhu, N.U. (1998). Stochastic Storage Processes, Springer, New York. 
[9] Sato. K. (1999). Léry Processes and Infinitely Divisible Distributions, Cambridge University Press, Cambridge.

[10] Takagi, H. (1991). Queueing Analysis. Vol. 1: Vacation and Priority Systems, Part 1, NorthHolland Publ. Co., Amsterdam. 